HStud 23 (2009)1, 29-35

DOI: 10.1556/HStud.23.2009.1.3

\title{
EIN BEITRAG ZUR KLÄRUNG DER FRAGE: WER WAR GOTTFRIED VON ROTTENSTEIN?
}

\author{
ANDREA SEIDLER \\ Universität Wien \\ Österreich
}

\begin{abstract}
Der Beitrag beschäftigt sich mit der Klärung der Identität des Pressburger Reiseschriftstellers Gottfried von Rottenstein, die in der zweiten Hälfte des 18. Jh. mehrere Beschreibungen seiner Reisen durch Europa herausgab und große Popularität erlangte. Die Historikerin Éva H. Balázs sah in ihm einen Abkömmling der Pálffy'schen Familie. Aus handschriftlichen Quellen konnte nun geklärt werden, dass es sich bei der Person um einen geadelten Bürgerlichen aus Pressburg handelt.
\end{abstract}

Schlüsselwörter: Aufklärung, Reisebeschreibung, Quellenforschung, Karl Gottlieb Windisch, Gottfried von Rottenstein, Geschichte Preßburgs

Die Identität Gottfried von Rottensteins (auch Rotenstein), eines Reiseschriftstellers, der in der zweiten Hälfte des 18. Jahrhunderts eine Reihe von Reisen durch deutsche Provinzen, durch Österreich und das Königreich Ungarn unternahm, beschäftigte nicht nur seine Zeitgenossen sondern auch Literaturwissenschaftler und Historiker. ${ }^{1}$ Eine Zusammenfassung seiner Reiseeindrücke erschien 1783 und 1792 in Leipzig in vier Teilen. ${ }^{2}$ Rottensteins Werke zeichneten sich nicht unbedingt durch rhetorische Spannung aus. Er beschrieb sämtliche bereisten Orte mit äußerster Präzision, gab detaillierteste Angaben zur Größe der Städte, der gesamten baulichen Anlage, der Einwohnerzahl sowie der Anzahl der Häuser. Die Darlegungen konzentrieren sich generell auf die Bewunderung von Wohlstand und Prunk, auf statistische und demografische Angaben - die Lektüre ist trocken, der ästhetische Anspruch fehlt gänzlich.

Über Wien fasste er beispielsweise zusammen:

Die Stadt Wien hat im Umfang 5 viertel Stunden, aber mit den Vorstädten, deren 32 sind, 4 gute Stunden. Es beträgt in der Länge 2 Stunden, in der Breite anderthalb Stunden. Sie hat ferner 6 Thore, 12 Bastionen, 68 Kirchen und Kapellen und vor jetzo in der Stadt 1344 von Stein gebaute Häuser, darunter 58 Paläste; mit den Vorstädten sind in allen 5500 Häuser... ${ }^{3}$ 
Die Einwohnerzahl gab Rottenstein mit 268.000 an, worunter 7000 Bürger gewesen sein sollen. Er wusste über die Finanzierung des Hauses der Verlegerfamilie Trattner ebenso Bescheid, ${ }^{4}$ wie über die Geschenke, die der marokkanische Gesandte Said Muhamad Ben Abdulmelek Pasa, vom 28. Februar 1783 bis 22. April 1783 marokkanischer Spezialgesandter in Wien, anlässlich seines Aufenthaltes vom Kaiser erhielt. ${ }^{5}$ Auffallend ist seine genaue Kenntnis der Hofburg, die er bis in die Einrichtung der Schlafzimmer des Kaiserpaares beschrieb:

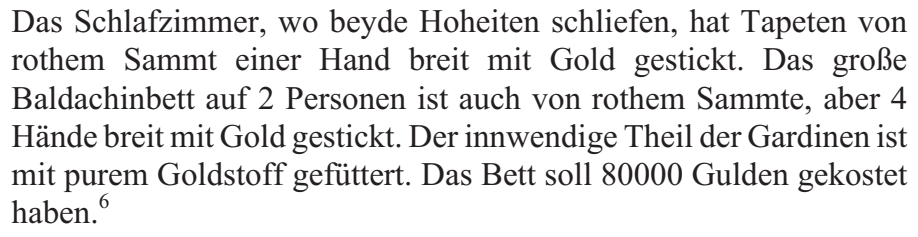

Seine Reise führte Rottenstein nach Pressburg. Auch von der ungarischen Hauptstadt gibt er dem Leser genaue Beschreibungen hinsichtlich der Größe, der Einwohner, der Anzahl der Häuser und mehr:

Pressburg, die schönste Stadt in Ungern, eine mittelmäßig große Stadt, mit einem Schloß; wenn man aber die Vorstädte dazuzählt, ein großer Orth, ${ }^{7}$

zeigt er sich begeistert.

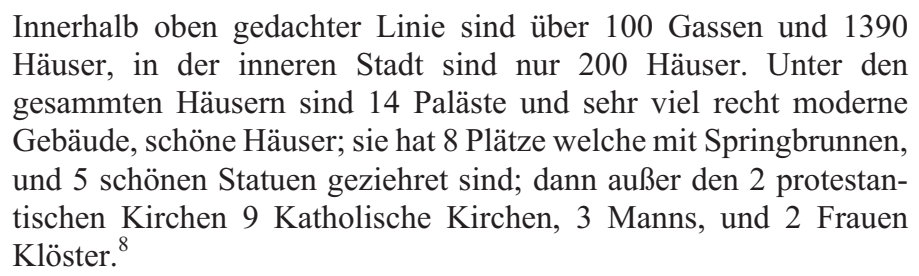

Die Einwohnerzahl gibt der Reisende mit 28.000 Personen, mit Vororten 36.109 an, davon seien 542 Edelleute, 1699 Bürger, 790 Geistlich und $200 \mathrm{Ju}-$ den. ${ }^{9}$ Er trug zweifelsohne einiges an wertvollen Informationen über seine Heimat zusammen.

Rottenstein muss auch den Privatgelehrten und Kommunalpolitiker Karl Gottlieb Windisch gekannt haben. Windisch war der Verfasser mehrerer historischer und geographischer Werke über das Königreich Ungarn und zeichnete für eine große Zahl von Zeitungs- und Zeitschriftengründungen in Pressburg verantwortlich. Um jene Zeit war er bereits über die Grenzen Ungarns bekannt und auch von der bedeutenden Allgemeinen Literatur-Zeitung wahrgenommen und positiv rezensiert worden. Der Text legt den Schluss nahe, dass Rottenstein sich im 
Windisch'schen Haus aufgehalten hat, denn auch hier beweist er wiederum genaue Kenntnis über die Bibliothek und die Ausstattung der Räume:

\begin{abstract}
In dem von Windischen Haus befinden sich einige sehr schöne Gemälde, und 2 sehr schöne Vasen von Volteranischem Alabaster. Sie sind mit erhabenen Weinranken unvergleichlich gezieret. Die Bibliothek ist zahlreich, und auserlesen; auch ist der Besitzer davon ein sehr gelehrter belesener Mann, welcher 5 Sprachen verstehet, und auch sehr viele nützliche Bücher fürs Vaterland geschrieben hat. Besonders eine vollständige Geographie von Ungern und Siebenbürgen. ${ }^{10}$
\end{abstract}

Er bewundert diesen Mann, der in der Folge nichts Gutes über ihn berichten wird! Rottenstein muss diese Beschreibung übrigens nach 1790 verfasst oder zumindest ergänzt haben, denn Windischs Geographie von Siebenbürgen erschien erst in nämlichem Jahr.

Die Reise führte ihn schließlich nach Pest, aber auch nach Esterhaß, zum prächtigen, erst in den 60er Jahren des 18. Jahrhunderts aufgebauten Schloss der fürstlichen Familie Esterházy. Auch hier fällt - wie schon zuvor - seine genaue Ortskenntnis vor allem in Bezug auf die Einrichtungen der Schlösser und Paläste sowie der in ihnen stattfindenden Festivitäten auf. Dieses genaue Wissen führte nicht nur zu seinen Lebzeiten zu Spekulationen hinsichtlich seiner Identität. Historiker beschäftigten sich gleichermaßen mit der Frage nach der Herkunft eines Mannes, der ihnen so ausführliche und wertvolle Beschreibungen dessen, was hinter verschlossenen Türen der Adeligen zu finden war, bot. Bernouilli, der Rottensteins Texte in die Sammlung von Reisebeschreibungen aufnahm, fühlte sich von diesen ausschließlich auf die Schilderung von Pomp und Reichtum ausgerichteten Aufzeichnungen irritiert und versuchte dem Leser die Arbeit dennoch schmackhaft zu machen. Er machte das Beste daraus und definierte den Wert des Textes über Informationen zum Wohlstand des Adels in einem unbekannten Land am östlichen Rand von Europa:

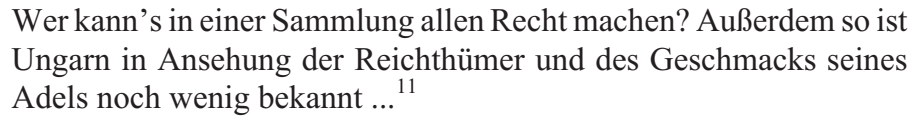

schrieb er in einer Vorrede zu der Publikation.

Auch die vor kurzem verstorbene Historikerin Éva H. Balázs versuchte in einer Abhandlung über Rottenstein dessen Identität zu erkunden. ${ }^{12}$ Sie stützte sich dabei auf diejenigen Textteile seiner Beschreibung, die sich mit Pressburg und der Umgebung der Stadt beschäftigen. Éva H. Balázs hält den Namen in der ausgeschriebenen Form, wie sie Bernouilli benutzte, nämlich Edler von Rottenstein, für ein Pseudonym, umso mehr, als sie in einschlägigen Lexika und Handbüchern keinerlei Hinweise auf dessen Identität festmachen konnte. Über die inhaltliche 
Analyse der Beschreibungen des Pálffyschen Schlosses in Königsaden (ung. Királyfa) und der Güter der Familie sowie die Ausführlichkeit in der Betrachtung der Feste auf dem Schloss Esterházy in Esterhaß (heute Fertőd) kommt sie zu dem Schluss, es handle sich bei Gottfried von Rottenstein um ein Mitglied der Familie Pálffy, genau gesagt um den Gardekapitän Johann Pálffy, 1744-1794:

\begin{abstract}
Somit haben wir in „Rottenstein“ einen Abkommen des „VizeKönigs“, dem Neffen des Kanzlers, dem Schwager des János Fekete, Haupt der ungarischen Opposition, dem Herrn von Biedersburg, der selbst Mitglied der Opposition war, eine für diese Epoche charakteristische widerspruchsvolle Persönlichkeit kennengelernt. ${ }^{13}$
\end{abstract}

Zunächst lässt sich die Argumentationskette gut nachvollziehen, die Éva H. Balázs zu ihrem Schluss führte: die allzu genaue Kenntnis der Schlösser und Paläste und die Hinweise auf Aufenthalte des Reiseschriftstellers auf den beschriebenen Gütern um Pressburg machen den Leser hellhörig und man vermutet, dass sich hinter dem Namen ein ortskundiger Adeliger verbergen muss. Auch der Heraldiker und Historiker Daniel Cornides, ein enger Mitarbeiter von K.G. Windisch, der die Reisebeschreibungen offensichtlich kannte, stellte sich die Frage nach der Identität Rottensteins und fragte bei Windisch als ebenfalls ortskundigen Pressburger an, der schließlich persönlich in Rottensteins Beschreibung verewigt worden war. Die Antwort Windischs war folgende:

Rottenstein ist ein bürgerl[icher] Apotheker, hat seinen Schild zum rohten Krebs, und seine Offizin auf dem Krautmarkte. Ein Erznarr, der unter allen Neunundreizigern in allen Kaiserlichen Erbländern, gewiß nicht seines Gleichen hat. - Vor ein par Jahren kaufte er sich den Oesterreichischen Adel, und heißt sonst Gottfried Stegmüller. Sein Vater war ein Bestandmüller in Pösching; und starb in der größten Dürftigkeit. Sein Sohn, unser geadelte Apotheker lernte sein Metier hier in Preßburg, dann als Geselle in Oedenburg, und Kaschau, heiratete die Tochter des Oedenburger Pfar[errs] Eitelhuber und kaufte mit seines Weibes, eines dummen Thiers, Golde, die Apotheke seines Lehrherren. - Da er als Gesell nicht reisen konnte, so that er es als Meister, und gab seine Reisen in Leipzig heraus, die in der Allgem[einen] Litt[eratur] Zeitung verdient gestrigelt wurden und deren auch die Ofner Blätter erwähnten. - Er ist das Gelächter der ganzen Stadt, träumt nur von Reisen, und seinem Adel, und ist zu dumm es zu merken, daß ihn jedermann zum Beßten hat. ${ }^{14}$

Durch die Kenntnis dieses Briefes drängt sich die Vermutung auf, dass Rottenstein tatsächlich mit dem geadelten Gottfried Stegmüller identisch sein könnte, der - zu Geld und Adel gekommen - Zugang zu den Adelshöfen oder zumindest zu ausführlichen Informationen darüber gehabt haben mag. Dies dürfte aufgrund der Öffnung der Prachtschlösser und das Einbeziehen des Publikums in die Schau 
der Reichen nicht schwer gewesen sein. Rottenstein schreibt beispielsweise über den Fürsten Nikolaus I. Esterházy und dessen Gastfreundschaft folgendes:

Der Fürst, ist ein Herr von mittelmäßiger Statur, hat ein schönes, männliches Gesicht und besitzt ein gutes Herz; er ist ein Kenner der Wissenschaften, und liebt die Künste und Musik; seine Lieblingsunterhaltungen sind die Musik und Opern... Das Bauen, die Lektür, sind auch Sache, die dieser Herr liebt; überhaupt hat er einen herrlichen Geschmack. [...] Seine Uniform, welche roth und mit Silber stark besetzt, ist mit Perlen gestickt; die Knöpfe mit Brillanten, die Gürtel mit Perlen, und der Reigerbusch auf der ungarischen Haube mit Smaragden und Diamanten besetzt. Des Pferdes Schabracke ist auch mit Perlen und das ganze Reitzeug mit Edelsteinen besetzt; dieser Schmuck wird über eine Million Gulden geschätzt. Ausser dem besitzt er einen schönen brillantenen Ring, wo der Hauptstein 30000 Fl. gekostet hat, [...] Sonst gehet dieser Herr Ganz simpel, deutsch aber gustos gekleidet, ist freundlich zu jedermann, lässt auch einen jeden hônnet-homme (welches auch mir einige Male wiederfahren) mit seinem Wagen in Esterhaz herumführen, und alle Merkwürdigkeiten zeigen. Die Opern und Comödien sind frey, so oft fremde kommen, und niemand gehet von Esterhaz weg, der nicht von der Freundlichkeit des Fürsten bezaubert ist. ${ }^{15}$

Jeden hônnet-homme also: und dazu zählt auch ein geadelter von Rottenstein, es muss nicht unbedingt ein Graf Pálffy sein.

Windisch weist in seinem Brief auf die Rezension einer Reisebeschreibung Rottensteins hin: verdient gestrigelt, also kritisiert habe man ihn in Deutschland. Diese Bemerkung bezieht sich vermutlich auf einen Beitrag in der Allgemeinen Literatur-Zeitung von dem März 1785, in dem der Rezensent gerade den oben dargelegten, langatmigen Stil des Verfassers als irritierend und überflüssig kritisiert.

Der Verfasser beschreibt hier umständlich die kaiserlichen Lustschlösser, Belvedere, Schönbrunn, Entzersdorf, Laxenburg, Hetzendorf, St. Veit, mit ihren Gärten, Gemälden, Statüen und anderen vorzüglichen Merkwürdigkeiten, [...] Was der Verfasser [...] vom Schlafzimmer des Kaisers erzählt, dass dieses africanisch mit Blumen gemahlt ist, könnte billig in einer Anmerkung erkläret werden. ${ }^{16}$

Wer verbirgt sich nun hinter Rottenstein? Pálffy oder Stegmüller?

Die Annahme Éva H. Balázs', dass es sich dabei um einen Grafen aus dem Pálffyschen Haus handelt, ist auf jeden Fall unrichtig. Windisch kannte die Schriftstellerszene seiner Heimatstadt sehr gut, er kannte Rottenstein persönlich und auch seinen familiären Hintergrund. Seine Informationen zu der Identität des Reiseschriftstellers sind richtig: das Adelslexikon verzeichnet nämlich zudem be- 
reits im Jahre 1822 die 1779 erfolgte Verleihung eines Adelstitels an den Pressburger Apotheker Stegmüller, der fortan den Namen von Rottenstein tragen sollte. ${ }^{1}$

In Kenntnis all dieser Quellen kann als gesichert gelten, dass die Identität Gottfried von Rottensteins geklärt ist und es sich bei seiner Person um den geadelten Apotheker Gottfried Stegmüller aus Pressburg handelt.

Windisch selbst besaß übrigens ein Exemplar der Reisebeschreibung Rottensteins. Jozef Tancer hat sie in seiner Bestandsaufnahme der Bibliothek des Privatgelehrten unter der Nummer 1093 verzeichnet. ${ }^{18}$

\section{Anmerkunden}

1 Gottfried von Rottenstein: Lust-Reise durch Bayern, Württemberg, Pfalz, Sachsen, Brandenburg, Österreich, Mähren, Böhmen und Ungarn in den Jahren 1784-1791. Leipzig: Schneider 1792-1793. 1782 und 1783 waren in Berlin bereits Werke Rottensteins erschienen: H.G.E.v.R. Lustreise durch Österreich und Mähren nach Brünn im September. Berlin, 1782 sowie ders. Reise von Wien nach Böhmen und Sachsen im May 1783. Berlin, 1783. ders. Beschreibung der Insel Schütt in Ungarn. Berlin, 1784. Diese Texte erschienen in der Sammlung: Bernouilli, Johann: Johann Bernouilli's Sammlung kurzer Reisebeschreibung und andere zur Erweiterung der Länder- und Menschenkenntniß dienender Nachrichten. Berlin: G. F. Richter und Buchhandlung der Gelehrten, 1781-1787, 17 Bde.

2 Vorliegende Arbeit stützt sich auf die Ausgabe 1792, die eine Zusammenfassung der Reiseeindrücke Rottensteins in den vorhergehenden acht Jahren bot.

3 Rottenstein, S. 82

4 „Das edle von Trattnersche Haus, des berühmten Buchhändlers und Buchdruckers, ist 5 Stock hoch und hat 14 Fenster in einer Reihe und 2 große Thore mit Balkons. Die Ballustrade des Daches ist mit dreizehn Statuen besetzet, in deren Mitte ein doppelter Adler, unter welchem folgende Inschrift Labore et Favore. Dieses Haus trägt 30000 Gulden an Miethe ein, es soll 300000 Gulden zu erbauen gekostet haben. "Rottenstein, S. 138.

5 „Der maroccanische Gesandte bekam 1783 für sich zum Präsent an porcellainenem Geräthen allein $6000 \mathrm{Fl}$. Werth, und für den maroccanischen Kaiser selbst folgendes Gegengeschenk: als eine grüne mit Gold gestickte Pferdeequipage; 2 schwarz sammtene mit Gold gestickte brodierte Geschirre auf eine Pirutsche, das Pirutsch ist mit feinem grünen Tuch und goldenen Dressen sehr reich verbrämt, 2 Schimmel zu Zugpferden; einen Rappen, und einen Falben, als Reitpferd; für 6000 Fl. schönes Porcellan, und eine Silbervergoldete Spieluhr. " Rottenstein, S. 206f.

6 Rottenstein, S. 92.

7 Rottenstein, S. 35

$8 \quad$ Rottenstein, Teil 3, S. 36.

9 Rottenstein, Teil 3, S. 42.

10 Rottenstein, Teil 3, S. 40; zur für seine Zeit tatsächlich auffallend reichhaltigen Bibliothek Windischs jüngst erschienen: Tancer, Jozef: Im Schatten Wiens. Zur deutschsprachigen Presse und Literatur im Pressburg des 18. Jahrhunderts. Bremen: edition lumière 2007.

11 Bernouilli, Band X, S. 186.

12 Siehe dazu die Studie von H. Balázs, Éva: Wer war Rottenstein? In: Mitteilungen des Österreichischen Staatsarchivs. Wien: 1990, Nr. 41, S. 43-52.

13 H. Balázs, Wer war Rottenstein?, S. 52. 
14 Seidler, Andrea (Hg.): Briefwechsel des Karl Gottlieb Windisch. Budapest: Universitas 2008; Magyarországi tudósok levelezése: Windisch an Cornides, 30. März 1787.

15 Rottenstein: Reisen durch einen Theil des Königreichs Ungern, im 1763sten und folgenden Jahren. In: Bernouilli: Sammlung kurzer Reisebeschreibungen, Band 9, 1783, S. 286.

16 Allgemeine Literatur-Zeitung vom Jahre 1785. Erster Band. Januar, Februar, März. Jena und Leipzig, 1785, März, S. 218.

17 Megerle von Mühlfeld, Johann Georg (Hg.): Österreichisches Adels-Lexikon des 18. und 19. Jahrhunderts: enthaltend alle von 1701 bis 1820 von den Souveränen Österreichs [...] in die verschiedenen Grade des deutsch-erbländischen oder Reichs-Adels erhobenen Personen. Wien, 1822, S. 268.

18 1093. Rottenstein v. [Rotenstein, Gottfried Edler von]: Reisen [Lustreisen durch Bayern, Würtemberg, Pfalz, Sachsen, Brandenburg, Oesterreich, Mähren, Böhmen und Ungarn, in den Jahren 1784 bis 1791. 3. Thle. Leipzig: Schneider 1792-1793]. Leipzig 1787, 8. In: Tancer, Im Schatten, Anhang. 
